\title{
Respiratory bronchiolitis in smokers with spontaneous pneumothorax
}

\author{
V. Cottin*, N. Streichenberger**, J-P. Gamondès***, F. Thévenet***, R. Loire**, J-F. Cordier*
}

Respiratory bronchiolitis in smokers with spontaneous pneumothorax. V. Cottin, N. Streichenberger, J-P. Gamondès, F. Thévenet, R. Loire, J-F. Cordier. CERS Journals 1998. ABSTRACT: Respiratory bronchiolitis (RB) is defined by the accumulation of pigmented macrophages in the lumen and wall of respiratory and membranous bronchioles of smokers. The aim of this study was to determine whether spontaneous pneumothorax was associated with a high prevalence of $R B$.

Seventy-nine consecutive patients who underwent a surgical procedure (thoracotomy or thoracoscopy) for recurrence or persistence of primary spontaneous pneumothorax despite thoracic drainage were studied retrospectively.

RB was found in 70 of $79(88.6 \%)$ smokers operated for spontaneous pneumothorax. Associated interstitial pathological abnormalities were present in 53 of 79 cases $(67.1 \%)$. In nine patients, the pathological lesions were severe and resembled desquamative interstitial pneumonia. Emphysematous lesions were present in about onethird of the patients.

Although the possible pathophysiological consequences of respiratory bronchiolitis remain speculative, this study demonstrates the high prevalence of this pathological abnormality in patients with pneumothorax requiring surgical treatment. Eur Resir J 1998; 12: 702-704.
*Service de Pneumologie, **Laboratoire d'Anatomie Pathologique, and $* * *$ Service de Chirurgie Thoracique, Hôpital Cardiovasculaire et Pneumologique Louis Pradel, Université Claude Bernard, Lyon, France.

Correspondence: J-F. Cordier, Service de Pneumologie, Hôpital Cardiovasculaire et Pneumologique Louis Pradel, 69394 Lyon Cedex 03, France. Fax: 33472357653

Keywords: Interstitial lung disease pneumothorax respiratory bronchiolitis

Recieved: June 31997

Accepted after revision April 81998

Supported by grant HCL-PNRC 93-005 and FRC-97 from Ministère de la Santé et de l'Action Humanitaire, France.
It has been well established that the risk of occurrence of spontaneous pneumothorax is linked to tobacco smoking, and increases with the length of exposure and the daily cigarette consumption [1]. However, how tobacco smoking promotes the development of pneumothorax is still unclear. It may induce pulmonary lesions, which in turn may promote the risk of pneumothorax [2].

Several pathological changes in the lung have been described in association with tobacco consumption, including disease of the large and small airways, pigment deposition and macrophage accumulation. Respiratory bronchiolitis (RB), described by NIEWOEHNER et al. [3], is defined by the accumulation of pigmented macrophages in the lumen and wall of respiratory and membranous bronchioles of smokers. The pigment deposition is considered to be related to tobacco smoking. Although RB was initially considered as an incidental finding, it has been suggested that it might contribute to the chronic small airway disease in smokers [3].

RB has not been studied in smokers with spontaneous pneumothorax. The aim of the present study was to determine whether spontaneous pneumothorax was associated with a high prevalence of tobacco-related RB on lung biopsy specimens of patients requiring surgical pleurodesis.

\section{Patients and methods}

\section{Patients}

One-hundred and eighteen patients underwent a surgical procedure for primary spontaneous pneumothorax, in- cluding a pulmonary biopsy, between 1978 and 1996 in the authors' hospital. Primary pneumothorax was defined by the exclusion of aetiological factors [2]. Fifteen patients were excluded because the pulmonary biopsy was too small to allow the study of bronchioles, 24 patients were nonsmokers and 79 patients were smokers and retained for the study. Indications for surgery were recurrent pneumothorax $(n=44)$, persistent air leak after 8 days of chest drainage or early recurrence after removal of the chest tube $(n=27)$, and/or prior controlateral pneumotho$\operatorname{rax}(n=8)$.

\section{Surgery}

Fifty patients underwent open surgical procedures and 29 videothorascopic procedures. The lungs were thoroughly inspected for blebs, bullae or air leaks, which were removed, together with the underlying peripheral part of the lung parenchyma (about $1 \mathrm{~cm}$ thick), and conserved for histological examination. Pleurodesis was obtained by parietal pleural abrasion using dry gauze $(n=26)$, apical pleurectomy $(\mathrm{n}=66)$, talc insufflation $(\mathrm{n}=7)$ and/or biological glue $(n=21)$.

\section{Histological examination}

The specimens were embedded in paraffin. The blocks were cut in 1-2 $\mu \mathrm{m}$ sections and stained with haematoxylin-phloxine-saffron for light microscopy. Perls's staining was used when the type of deposited pigments (haemosiderin or tobacco-associated pigments) was in doubt. From 
each specimen the following histological features were assessed: number of respiratory bronchioles present in the sample, RB, inflammatory cell infiltration or fibrotic changes to the airway wall and interstitial spaces, pigment deposition and concomitant emphysematous lesions.

$\mathrm{RB}$ was defined [3] by the accumulation of pigmented macrophages in the lumen and wall of respiratory bronchioles (obligatory), and in neighbouring alveolar ducts and alveoli, associated with submucosal and peribronchiolar infiltration by inflammatory cells in adjacent bronchiolar walls. Severity of RB was graded according to the extent of pigmented macrophage deposition around the respiratory bronchiole: grade 1 was defined by the accumulation of pigmented macrophages limited to the lumen and wall of respiratory and membranous bronchioles (at least one bronchiole involved) and to a single-layer ring of peribronchiolar alveoli; grade 2 was defined as the extent of the deposition of tan-brown macrophages in the adjacent alveolar ducts and alveoli; grade 3 was defined as a massive deposition of pigmented macrophages in the peribronchiolar area, with RB not involving all the bronchioles of the sample; and grade 4 was defined as a massive involvement of bronchiolar and alveolar lumen in the whole sample. Concomitant interstitial lung disease (ILD) was defined by an excess of connective tissue in the interstitium and/or interstitial inflammatory cell infiltration [4, 5]. The concomitant presence of emphysema was also investigated, and it was further classified into centrilobular, panlobular and localized (paraseptal) emphysema.

\section{Results}

\section{Patient characteristics}

The patients were 63 males and 16 females, aged $40 \pm 12$ yrs (range 17-71). Mean height was $173 \pm 9 \mathrm{~cm}(152-197)$, weight $62 \pm 11 \mathrm{~kg}(42-97)$ and body mass index $20.4 \pm 2.5$ $\mathrm{kg} \cdot \mathrm{m}^{-2}(15.6-28)$. All were smokers with a mean smoking history of $13.8 \pm 12.6$ pack-yrs (1-60). $\alpha 1$-Antitrypsin level, available for 17 patients, was within the normal range $(2.3 \pm$ $0.7 \mathrm{~g} \cdot \mathrm{L}^{-1}$, range $\left.1.0-3.4\right)$. Pulmonary function tests were systematically performed for 29 patients, 4-10 weeks after the surgical procedure, and were within the normal range.

\section{Perioperative findings}

Perioperative macroscopic examination of the lung revealed active air leak in 11 cases, subpleural bullae in 74 cases and pleural adhesions in 22 cases.

\section{Histological findings of lung tissues}

RB was found in 70 of 79 smokers operated for spontaneous pneumothorax (88.6\%). The grading of RB is shown in table 1 . Histological RB-associated ILD was present in 53 of 79 cases $(67.1 \%)$. The main interstitial abnormality associated with RB was a thickening of the interstitial structures, consisting of an excess of connective tissue. Inflammatory cell infiltration of the alveolar and interlobular septa, the peribronchiolar zone and the visceral pleura
Table 1. - Histological findings of lung tissues

\begin{tabular}{lc}
\hline & $\begin{array}{c}\text { Patients } \\
\mathrm{n}(\%)\end{array}$ \\
\hline Number of bronchioles per sample & $4.4 \pm 2.4$ \\
Respiratory bronchiolitis & $21(30.0)^{\dagger}$ \\
Grade 1 & $16(22.9)^{\dagger}$ \\
Grade 2 & $22(31.4)^{\dagger}$ \\
Grade 3 & $11(15.7)^{\dagger}$ \\
Grade 4 & $70(88.6)$ \\
Overall & $53(67.1)$ \\
Interstitial abnormalities & $41(51.9)$ \\
Inflammatory cell infiltration & $45(57.0)$ \\
Fibrosis & \\
Pigments & $70(88.6)$ \\
Tobacco-associated pigments & $15(19.0)$ \\
Anthracosis & $9(11.4)$ \\
Haemosiderin & \\
Emphysema & $0(0)$ \\
Panlobular & $8(10.1)$ \\
Centrilobular & $7(8.9)$ \\
Paraseptal (localized) & $7(8.9)$ \\
Unclassified & $5(6.3)$ \\
Bullae & $27(34.2)$ \\
Overall & $31(39.2)$ \\
Arteriolar intimal fibrosis &
\end{tabular}

: Expressed as per cent of positive cases of respiratory bronchiolitis.

was also frequent. In nine patients, marked interstitial abnormalities were associated with massive deposition of pigmented macrophages involving all bronchiolar and alveolar lumens in the sample (RB grade 4); the pathological lesions in these patients resembled desquamative interstitial pneumonia. A mild thickening of the interstitial spaces without inflammatory infiltration was also noted in two patients without RB. Non-tobacco pigment deposition was markedly less frequent than tobacco-associated pigments (table 1). Emphysematous lesions were present in about one-third of the patients, but distinguishing between different classes of emphysematous lesions was difficult because of the small size of many surgical samples. Surprisingly, a marked intimal fibrosis of the small pulmonary arterioles was frequently noted (39\%).

\section{Discussion}

Although RB may be a common finding in healthy smokers, its precise prevalence is not known. In the present study RB was found in nearly $90 \%$ of lung biopsy specimens from smokers requiring surgical pleurodesis for spontaneous pneumothorax. Since the specimens for histological examinations consisted of the area of lung parenchyma adjacent to the resected lesions and RB occurs in an irregular distribution [3] this prevalence may even be underestimated.

In a subset of patients, RB is associated with ILD and manifests with dyspnoea, pulmonary function and radiological abnormalities [4-6]. In the present study, RB was frequently associated with thickening of the interstitial spaces, together with inflammatory cell infiltration of the alveolar and interlobular septa and the peribronchiolar zone. In these patients with ILD, RB lesions were more pronounced, as reported previously [5, 7], and resembled 
desquamative interstitial pneumonia, an entity distinct from RB. Accumulation of intra-alveolar macrophages and mild interstitial thickening occur in both conditions, but in RB the process is patchy and follows a peribronchiolar distribution [8].

Although a high prevalence of $\mathrm{RB}$ in patients with spontaneous pneumothorax has been shown by the present study, its pathophysiological significance remains to be clarified. Peripheral airway lesions such as RB may be the precursor of more severe anatomical lesions such as centriacinar emphysema [9]. The tobacco-induced chronic inflammatory reaction may spread centrifugally from the small airways to the adjacent parenchyma and lead to progressive destruction of peribronchiolar alveoli [10]. Some support for this concept has come from the demonstration of a relationship between bronchiolar disease and centriacinar emphysema in previous pathological studies $[6,9$, $11,12]$. In addition, physiological studies have shown a link between tobacco-associated bronchiolar disease such as RB and functional abnormalities of the small airways $[13,14]$. A similar mechanism may be involved in the pathogenesis of subpleural bullae and blebs in smokers, lesions frequently associated with idiopathic spontaneous pneumothorax [15]. Whether tobacco-induced pathological changes in the small airways including $\mathrm{RB}$ might contribute to the development of focal emphysema with consequent formation of bullae, thus predisposing to spontaneous pneumothorax, remains to be studied.

Although the possible pathophysiological consequences of respiratory bronchiolitis remain speculative, this study demonstrates the high prevalence of this pathological abnormality in patients with pneumothorax requiring surgical treatment.

Acknowledgment: The authors are indebted to T. Greenland, for critical review of the manuscript.

\section{References}

1. Bense L, Eklund G, Wiman LG. Smoking and the increased risk of contracting spontaneous pneumothorax. Chest 1987; 92: 1009-1012.

2. Light RW. Pneumothorax. In: Murray JF, Nadel JA, eds.
Textbook of Respiratory Medicine. Philadelphia, PA, WB Saunders, 1994; pp. 2193-2210.

3. Niewoehner DE, Kleinerman J, Rice DB. Pathologic changes in the peripheral airways of young cigarette smokers. N Engl J Med 1974; 291: 755-758.

4. Myers JL, Veal CF, Shin MS, Katzenstein AL. Respiratory bronchiolitis causing interstitial lung disease. A clinicopathologic study of six cases. Am Rev Respir Dis 1987; 135: 880-884.

5. Yousem SA, Colby TV, Gaensler EA. Respiratory bronchiolitis-associated interstitial lung disease and its relationship to desquamative interstitial pneumonia. Mayo Clin Proc 1989; 64: 1373-1380.

6. Kim WD, Eidelman DH, Izquierdo JL, Ghezzo H, Saetta MP, Cosio MG. Centrilobular and panlobular emphysema in smokers: two distinct morphologic and functional entities. Am Rev Respir Dis 1991; 144: 1385-1390.

7. King TE Jr. Respiratory bronchiolitis-associated interstitial lung disease. Clin Chest Med 1993; 14: 693-698.

8. Katzenstein ALA, Askin FB. Idiopathic interstitial pneumonia/idiopathic pulmonary fibrosis. In: Katzenstein ALA, Askin FB, eds. Surgical Pathology of Non-neoplastic Lung Disease. Philadelphia, PA, WB Saunders, 1990; pp. 58-96.

9. Leopold JG, Gough J. The centrilobular form of hypertrophic emphysema and its relation to chronic bronchitis. Thorax 1957; 12: 219-235.

10. Finkelstein RA, Cosio MG. Disease of the small airways in smokers: smokers' bronchiolitis. In: Epler GR, ed. Diseases of the Bronchioles. New York, Raven Press, 1994; pp. 115-137.

11. Cosio MG, Hale KA, Niewoehner DE. Morphologic and morphometric effects of prolonged cigarette smoking on the small airway. Am Rev Respir Dis 1980; 122: 265-271.

12. Willems LNA, Kramps JA, Stijnen T, Sterk PJ, Weening JJ, Dijkman JH. Relation between small airways disease and parenchymal destruction in surgical lung specimens. Thorax 1990; 45: 89-94.

13. Cosio M, Ghezzo H, Hogg JC, et al. The relations between structural changes in small airways and pulmonary-function tests. N Engl J Med 1978; 298: 1277-1281.

14. Wright JL, Lawson LM, Paré PD, Kennedy S, Wiggs B, Hogg JC. The detection of small airways disease. Am Rev Respir Dis 1984; 129: 989-994.

15. Jordan KG, Kwong SK, Flint J, Müller NL. Surgically treated pneumothorax. Radiologic and pathologic findings. Chest 1997; 111: 280-285. 